\title{
Freie Gelenkkörper als Unruhestifter
}

\author{
Selten, aber schmerzhaft. Wenn \\ sich die ersten Symptome \\ zeigen, ist die Chondromatose \\ meist schon fortgeschritten. Sehr \\ selten kann es zu einer malignen \\ Transformation in ein sekundä- \\ res Chondrosarkom kommen.
}

— Das Auftreten freier Gelenkkörper im Glenohumeralgelenk ist, im Vergleich zu den anderen größeren Gelenken, relativ selten $[1,2,8,10,11]$. Die Ursache ist i.d.R. eine synoviale Chondromatose des Gelenks, der Bursa subacromialis, eine Osteochondrosis dissecans oder Omarthrose.

Die primäre synoviale Chondromatose ist charakterisiert durch eine primär benigne kartilaginäre Metaplasie synovialer Membranen. Knie-, Ellenbogen- und Hüftgelenke sind am häufigsten betroffen. Es entstehen multiple freie Gelenkkörper, die zur Kalzifizierung neigen. Die Ätiologie ist bis heute nicht geklärt. Im Gegensatz zur sekundären synovialen Chondromatose liegt bei der primären Form eine aktive Metaplasie und nicht nur eine Einbettung von Knorpelfragmenten in die Synovialmembran vor [11].

Die primäre synoviale Chondromatose wird in drei separate Phasen nach Milgram unterteilt [5]:

- Phase 1: aktiver intrasynovialer Prozess ohne freie Körper,

- Phase 2: Zwischenstadium mit aktiver intrasynovialer Proliferation und intraartikulären freien Körpern,

- Phase 3: freie Körper ohne nachweisbare intrasynoviale Veränderungen.

\section{Befund}

Aufgrund der initial geringen Symptome erfolgt die Diagnose meist erst in Phase 2. Dann liegen unspezifische Beschwerden wie Schmerzen, Bewegungseinschränkung, rezivivierende Blockierungen, Schwel-

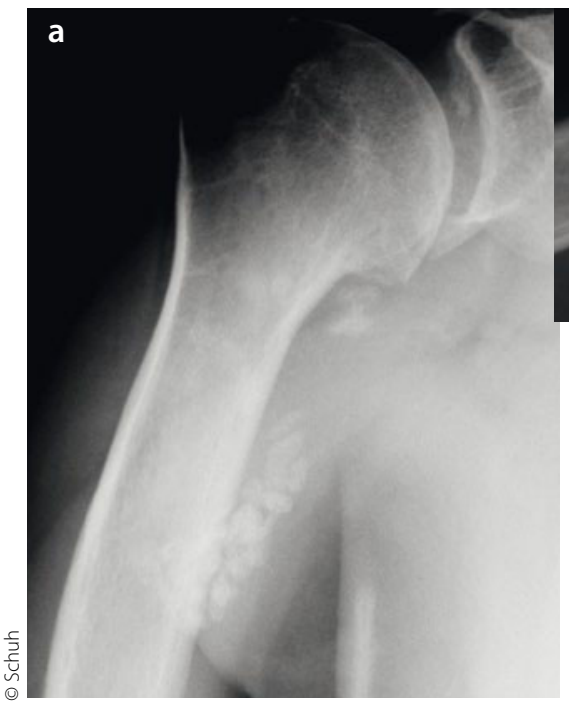

lungen synovialer Membranen, Krepitus und ggf. tastbare freie Körper vor $[1,2,11]$.

\section{Bildgebung}

Die radiologischen Befunde reichen vom Normalbefund bis zum Nachweis multipler röntgendichter freier Körper (Abb. 1), je nach Stadium der Erkrankung. Im Frühstadium können Kernspintomografie oder Doppelkontrast-CT die Körper bereits vor der Kalzifizierung darstellen [11].

\section{Differenzialdiagnose}

Als Differenzialdiagnose sind Tendinosis calcarea, Osteochondrosis dissecans, neurogene Arthropathie, chronische septische Arthritis, villonoduläre Synovitis, rheumatoide Arthritis und Synovialsarkome zu bedenken [11].

\section{Therapie}

Die Behandlung der Chondromatose erfolgt mittels arthroskopischer oder offener Entfernung der freien Körper und partieller, ggf. vollständiger Synovektomie [2, 4, 7, 9-11]. Dadurch wird bei noch bestehender metaplastischer Aktivität die Entstehung neuer Körper verhindert. Neben der geringeren Morbidität ist bei der Arthroskopie das komplette Gelenk einsehbar, was die Diagnostik und Therapie wei-

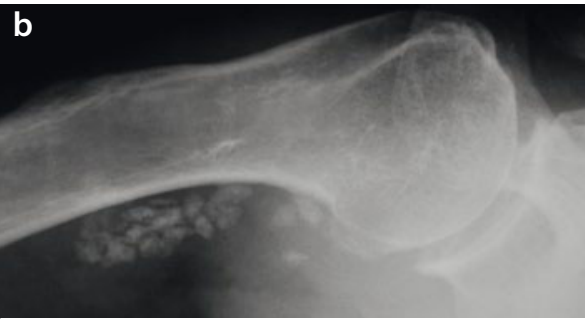

Abb. 1 Im Röntgenbild a.p. (a) und seitl. (b) der rechten Schulter zeigen sich multiple Verkalkungen, insbesondere im Recessus axillaris.

terer intraartikulärer Pathologien erlaubt. Daneben kann die Arthroskopie zur Diagnosesicherung bei primär unklaren Fällen dienen [6, 11]. Die Ergebnisse der Behandlung hängen vom Zeitpunkt der Intervention ab. Dies gilt insbesondere für die sekundäre Knorpelschädigung.

Bei der Indikation zu operativen Maßnahmen muss neben dem klinischen Ergebnis auch das Rezidiv- und Entartungsrisiko bedacht werden. Das relative Risiko eines Rezidivs der primären synovialen Chondromatose liegt bei 15\% [3]. Die schwerwiegendste Komplikation der primären synovialen Chondromatose ist eine maligne Transformation in ein sekundäres (synoviales) Chondrosarkom. In Langzeitstudien wurden Transformationsraten von bis zu 5\% beschrieben [3]. Zweifelsohne ist es schwierig hierbei zu differenzieren, ob es sich um eine echte maligne Transformation eines zunächst benignen Krankheitsbildes oder um die Fehldiagnose eines Low-grade-Synovialsarkoms zum Zeitpunkt der Erstuntersuchung handelt. .

\section{Literatur unter mmw.de}

Für die Verfasser:

PD Dr. med. habil. Alexander Schuh Akad. Lehrkrankenhaus der FriedrichAlexander-Universität Erlangen-Nürnberg Nürnberger Str. 12, D-92318 Neumarkt i.d. OPf. E-Mail:Alexander.Schuh@klinikum.neumarkt.de

Keywords

Chromatosis of the Shoulder Joint

Chromatosis - Shoulder joint 